\title{
Erythropoietin reduces brain injury after intracerebral hemorrhagic stroke in rats
}

\author{
ZHEN YU ${ }^{1}$, LING TANG ${ }^{2}$, LIFEN CHEN ${ }^{1}$, JINFANG LI ${ }^{1}$, \\ WANFU $\mathrm{WU}^{3}$ and CHANGLIN HU ${ }^{1}$ \\ ${ }^{1}$ Department of Neurology, The Second Affiliated Hospital; ${ }^{2}$ Department of Neurology, \\ The Fifth Affiliated Hospital; ${ }^{3}$ Department of Neurology, The First Affiliated Hospital, \\ Chongqing University of Medical Sciences, Chongqing 400010, P.R. China
}

Received April 1, 2013; Accepted August 5, 2013

DOI: $10.3892 / \mathrm{mmr} .2013 .1666$

\begin{abstract}
Erythropoietin (EPO) has been shown to be neuroprotective in various models of neuronal injury. The aim of the present study was to investigate the beneficial effect of recombinant human EPO (rhEPO) following intracerebral hemorrhage ( $\mathrm{ICH})$ and the underlying molecular and cellular mechanisms. ICH was induced using autologous blood injection in adult rats. rhEPO $(5000 \mathrm{IU} / \mathrm{kg})$ or vehicle was administered to rats with ICH $2 \mathrm{~h}$ following surgery and every $24 \mathrm{~h}$ for 1 or 3 days. To study the involvement of the PI3K signaling pathway in the rhEPO-mediated effect, the PI3K inhibitor wortmannin $(15 \mu \mathrm{g} / \mathrm{kg})$, was intravenously administered to rats with ICH 90 min prior to rhEPO treatment. Brain edema was measured 3 days following ICH and behavioral outcomes were measured at 1, 7, 14,21 and 28 days following ICH using the modified neurological severity score (mNSS) and the corner turn test. Proinflammatory cytokines, including tumor necrosis factor (TNF)- $\alpha$, interleukin (IL)-1 $\beta$ and IL-6, in the ipsilateral striatum were analyzed using an enzyme-linked immunosorbent assay $24 \mathrm{~h}$ following ICH. Neuronal apoptosis in the perihematomal area was determined by NeuN and terminal deoxynucleotidyl transferase dUTP nick end labeling (TUNEL) double-staining. The results showed that rhEPO treatment reversed ICH, increased brain water content, upregulated proinflammatory cytokines, neuronal loss and apoptosis in the perihematomal area and rescued behavioral deficits in injured rats. Inhibiting the PI3K pathway with wortmannin abolished the rhEPO-mediated neuroprotective effects. Moreover, western blot analysis showed that rhEPO induced the upregulation of Akt phosphorylation and downregulation of glycogen synthase
\end{abstract}

Correspondence to: Dr Zhen Yu, Department of Neurology, The Second Affiliated Hospital, Chongqing University of Medical Sciences, 76 Lin Jiang Men, Chongqing 400010, P.R. China E-mail: zhenyu4002@126.com

Key words: intracerebral hemorrhage, stroke, erythropoietin, PI3K signaling pathway kinase (GSK)-3 $\beta$ phosphorylation, which were reversed by pretreatment with wortmannin, indicating the involvement of PI3K signaling in rhEPO-mediated anti-apoptotic and anti-inflammatory effects following ICH. In conclusion, these results suggested that rhEPO may exert its beneficial effects in ICH through the activation of the PI3K signaling pathway.

\section{Introduction}

Intracerebral hemorrhage (ICH) constitutes $15 \%$ of all strokes in the USA and Europe, and 20-30\% in Asian and African populations (1). Approximately $30-40 \%$ of ischemic strokes undergo a certain degree of hemorrhagic conversion (2). ICH is associated with high mortality and disability. To date, surgical approaches for the treatment of ICH have not been particularly effective and no satisfactory drug treatment is currently used in clinical practice $(3,4)$.

ICH is a dynamic, serial process in which early hematoma expansion with excitotoxicity, perihematomal inflammation and apoptosis occurs (5). Consequently, prospective treatments reducing brain edema or apoptotic cell death may provide a neuroprotective strategy for ICH patients.

Erythropoietin (EPO) is a glycoprotein that is predominantly produced in the adult kidneys and the liver. EPO and the EPO receptor (EPOR) are essential for erythropoiesis and are also expressed in neurons, astrocytes and cerebral endothelial cells in the brain. EPO has been shown to exhibit anti-apoptotic, anti-inflammatory, anti-oxidative, angiogenetic and neurotrophic properties (6). Its potential therapeutic effect has been demonstrated in the animal model of cerebral ischemia (7-9), spinal cord injury $(10,11)$, traumatic brain injury $(12,13)$ and subarachnoid hemorrhage (14-16).

Recently, studies have demonstrated the beneficial involvement of EPO in ICH, possibly through its anti-inflammatory and anti-apoptotic effects (17-20). However, the molecular and cellular mechanisms and the signaling pathway underlying these effects remains to be elucidated.

The present study aimed to investigate the effect of rhEPO in an autologous blood-injected rat model of ICH and the underlying molecular and cellular mechanisms, particularly the involvement of the PI3K signaling pathway. 


\section{Materials and methods}

Animal preparation and ICH model. The rats were obtained from the Experimental Animal Center of the 3rd Military Medical University (Chongqing, China) and the animal protocol for this study was approved by the Institutional Animal Care and Use Committee of the Chongqing University of Medical Sciences (Chonqing, China). Adult male Sprague-Dawley rats ( $\mathrm{n}=64$; weight, 280-300 g) were used in this study. Animals were allowed access to food and water ad libitum prior to the experiments. The ICH model was performed as described previously (21). The rats were anesthetized with an intraperitoneal injection of $10 \%$ chloral hydrate at $400 \mathrm{mg} / \mathrm{kg}$ and positioned in a stereotaxic frame (David Kopf Instruments, Tujunga, CA, USA). Under sterile conditions, a cranial burr hole $(1 \mathrm{~mm})$ was drilled near the right coronal suture, $3.5 \mathrm{~mm}$ lateral to the midline. A 26 -gauge needle was inserted stereotaxically into the right basal ganglia (coordinates: $0.2 \mathrm{~mm}$ anterior, $5.5 \mathrm{~mm}$ ventral and $3.5 \mathrm{~mm}$ lateral to the bregma). Autologous whole blood (100 $\mu \mathrm{l}$, with no anticoagulants) was injected at a speed of $10 \mu \mathrm{l} / \mathrm{min}$ with a microinfusion pump (Braun, Melsungen, Germany). The needle was removed and the skin incision was sutured following infusion. All rats were allowed to recover fully under observation. Sham-operated animals were subjected to needle insertion only.

Experimental groups and pharmacological interventions. The rats were divided into the following four groups: Group 1, sham $(\mathrm{n}=18)$; group $2, \mathrm{ICH}+$ vehicle $(\mathrm{n}=18)$; group $3, \mathrm{ICH}+\mathrm{rhEPO}$ $(\mathrm{n}=18)$; and group 4, $\mathrm{ICH}+\mathrm{rhEPO}+$ wortmannin $(\mathrm{n}=18)$. $\mathrm{rhEPO}$ (H. Lundbeck A/S, Copenhagen, Denmark) was administered intraperitoneally at 2,24 and $48 \mathrm{~h}$ at $5000 \mathrm{IU} / \mathrm{kg}$ body weight following ICH. The experimental design is shown in Fig. 1. The animals in the ICH + vehicle group received vehicle intraperitoneally at 2,24 and $48 \mathrm{~h}$. The ICH rats received the PI3K inhibitor wortmannin $(15 \mu \mathrm{g} / \mathrm{kg})$ intravenously $90 \mathrm{~min}$ prior to rhEPO injection. The vehicle-treated animals received normal saline at a volume equivalent to that administered to animals in the intervention groups. All chemicals were purchased from Sigma-Aldrich (St. Louis, MO, USA) and processed according to previously published protocols $(22,23)$. The dosage of rhEPO was based on previous studies on traumatic brain injury and ischemic stroke $(19,12)$.

Assessment of behavioral outcome. Behavioral outcomes were measured using the modified neurological severity score (mNSS) (24) and the corner turn test score (25). These were performed by an observer who was blinded to the individual treatment status of the animals. Behavioral outcomes for each rat were tested 1, 7, 14, 21 and 28 days following $\mathrm{ICH}$. The mNSS is a composite of motor (muscle status and abnormal movement), sensory (visual, tactile and proprioceptive), reflex and balance tests. Neurological function was graded on a scale of 0 to 18 (normal score, 0 ; maximal deficit score, 18), where higher scores indicate more severe behavioral deficits. The corner test detects impairment of sensorimotor function. Briefly, one rat was placed between two boards that were attached with the edges at a $30^{\circ}$ angle to each other. The control rat turned either left or right, but the hemorrhagic rat preferentially turned toward the non-impaired side. The number of right turns was recorded from 10 tests for each rat.

Measurement of brain water content. The brain water content was measured 3 days following ICH injury. The rats were decapitated under deep anesthesia and the brains were immediately removed and cut into 4-mm sections. Each section was divided into four parts; ipsilateral and contralateral basal ganglia, and ipsilateral and contralateral cortex. The cerebellum was collected as the internal control. Tissue samples were weighed on an electronic analytical balance (Changzhou Instruments, Changzhou, China) to the nearest $0.1 \mathrm{mg}$ to obtain the wet weight. The brain samples were then dried at $100^{\circ} \mathrm{C}$ in an electric blast-drying oven (Sida Apparatus, Jiangsu, China) for $24 \mathrm{~h}$ to obtain the dry weight. The brain water content (\%) was calculated as (wet weight - dry weight)/wet weight $\mathrm{x} 100$.

Determination of inflammatory cytokines in hemorrhagic brain tissue. To determine the levels of inflammatory cytokines in the striatal tissue, ipsilateral striatal tissues were collected $24 \mathrm{~h}$ following ICH. The tissues were lysed $(40 \%$, $\mathrm{wt} / \mathrm{vol}$ ) with $0.01 \mathrm{~mol} / \mathrm{l}$ phosphate-buffered saline ( $\mathrm{pH} 7.4)$ containing a protease inhibitor cocktail (Roche, Indianapolis, IN, USA) and homogenized. The homogenates were then centrifuged at $7500 \mathrm{x} \mathrm{g}$ for $20 \mathrm{~min}$ at $4^{\circ} \mathrm{C}$ using a highspeed cryostat centrifuge (3K30; Sigma Centrifuges $\mathrm{GmbH}$, Osterode am Harz, Germany). The levels of tumor necrosis factor- $\alpha$ (TNF- $\alpha$ ), interleukin (IL)- $1 \beta$ and IL- 6 in the supernatant were determined by enzyme-linked immunosorbent assay (ELISA) using commercial kits (DuoSetELISA Development Systems; R\&D Systems, Minneapolis, MN, USA) according to the manufacturer's instructions via an ELISA analyzer (Coda, Bio-Rad, Hercules, CA, USA).

Immunofluorescent staining. The rats were euthanized $24 \mathrm{~h}$ following surgery and brain specimens were processed for immunostaining as previously described (1). Double immunofluorescence staining was performed using the neuronal marker anti-NeuN monoAb (1:100, Millipore, Temecula, CA, USA) and terminal deoxynucleotidyl transferase dUTP nick end labeling (TUNEL; Roche). Once stained, the specimens were analyzed under a fluorescence microscope (Olympus BX51; Olympus, Melville, NY, USA). The TUNEL-positive cells were counted at the center of the hemorrhagic lesion. Three perihematomal regions of the ipsilateral cerebral hemisphere were used for cell counting. The result was presented as the cell density of TUNEL-positive or TUNEL/NeuN-positive cells. Four rats per group were used for intergroup comparisons.

Western blot analysis. Rats were sacrificed $24 \mathrm{~h}$ after ICH and western blot analysis was performed as previously described (26). The brain samples were homogenized and the total proteins were extracted using radio immunoprecipitation assay lysis buffer (Beyotime Biotech. Co., Nanjing, China). Samples with an equal quantity of protein $(50 \mu \mathrm{g})$ were separated on $10 \%$ sodium dodecyl sulfate polyacrylamide gels, transferred onto nitrocellulose membranes and blocked in 5\% non-fat dry milk buffer at $4^{\circ} \mathrm{C}$ overnight. The membranes were then incubated overnight at $4{ }^{\circ} \mathrm{C}$ with monoclonal rabbit anti-phospho-glycogen synthase kinase (GSK)-3 $\beta$ (Ser9; 1:1,000; Cell Signaling Technology,Inc.,Beverly, 


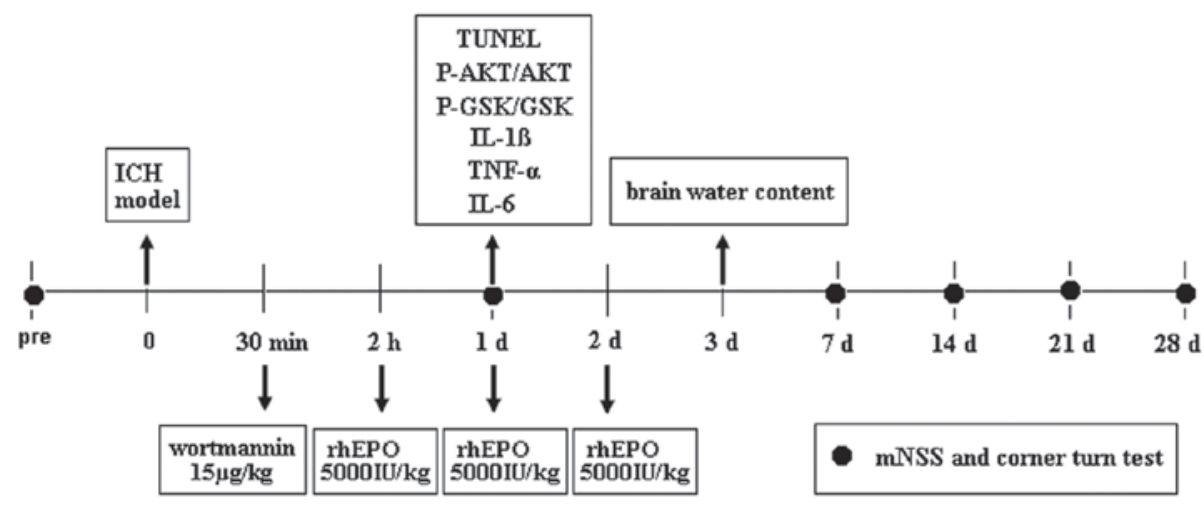

Figure 1. Experimental design. ICH, intracerebral hemorrhage; rhEPO, recombinant human erythropoietin; mNSS, modified neurological severity score; TUNEL, terminal deoxynucleotidyl transferase dUTP nick end labeling; p-AKT, phosphorylated-AKT; GSK, glycogen synthase kinase; IL, interleukin; TNF- $\alpha$, tumor necrosis factor- $\alpha$.

MA, USA), monoclonal rabbit anti-total GSK-3 $\beta$ (1:2,000; Cell Signaling Technology, Inc.), polyclonal rabbit anti-phospho-Akt (Ser473; 1:1000; Cell Signaling Technology, Inc.) and polyclonal rabbit anti-Akt (1:1,000; Cell Signaling Technology, Inc.) for $1 \mathrm{~h}$, incubated with Enhanced Chemiluminescence Plus solution (GE Healthcare, Little Chalfont, UK) for $5 \mathrm{~min}$ and were densitometrically quantified and analyzed using an NIH image analysis system. Phosphorylation levels of protein were indicated by the ratio of phospho-protein to total protein. The value was then normalized to the mean value of the sham-operated group for comparison.

Statistical analysis. The data are expressed as the mean \pm SEM and were statistically analyzed using one-way analysis of variance (ANOVA) followed by Tukey's post hoc test. All behavioral data are expressed as the mean \pm SEM of sham performance and analyzed with Kruskal-Wallis one-way ANOVA on ranks, followed by the Student-Newman-Keuls method. $\mathrm{P}<0.05$ was considered indicate a statistically significant difference. All statistical analyses were performed using SigmaPlot version 10.0 (Systat Software, Inc., San Jose, CA, USA) for Windows.

\section{Results}

rhEPO attenuates brain edema 72 following ICH. Brain edema was evaluated 3 days following $\mathrm{ICH}$ ( $\mathrm{n}=6$ per group). Rats with intracerebral hemorrhage showed significant brain edema in the ipislateral tissue compared with the sham group $(\mathrm{P}<0.05)$. rhEPO treatment significantly reduced brain water content in the ipsilateral basal ganglia and cortex in hemorrhagic rats compared with rats treated with vehicle $(\mathrm{P}<0.05$, Fig. 2). However, administration of the PI3K inhibitor wortmannin $(15 \mu \mathrm{g} / \mathrm{kg})$ prior to rhEPO treatment completely abolished its effect $(\mathrm{P}<0.05$ compared with rhEPO) without exacerbating brain edema in hemorrhagic rats. No significant differences were identified in the contralateral cortex, contralateral basal ganglia or cerebellum among all groups.

rhEPO attenuates behavioral deficits at days 7 to 28 following $\mathrm{ICH}$. Rats from the sham group showed no neurological deficits at any time-point. Day 1 post-ICH, all rats with ICH showed significantly increased scores in $\operatorname{mNSS}(\mathrm{P}<0.05$,

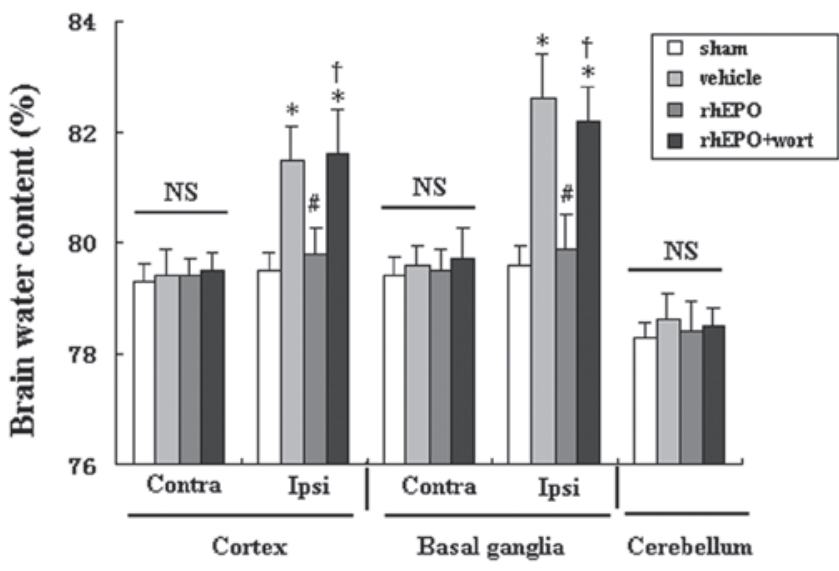

Figure 2. Measurement of brain edema 3 days following ICH or sham operation. Data are expressed as the mean \pm SEM. ${ }^{*} \mathrm{P}<0.05$ vs. the sham-operated rats, ${ }^{\#} \mathrm{P}<0.05$ vs. the vehicle-treated rats and ${ }^{\dagger} \mathrm{P}<0.05$ vs. rhEPO-treated rats ( $n=6$ per group). contra, contralateral; ipsi, ipsilateral; NS, not significant; $\mathrm{ICH}$, intracerebral hemorrhage; rhEPO, recombinant human erythropoietin.

Fig. 3A) and the corner turn test $(\mathrm{P}<0.05$, Fig. 3B), suggesting that $\mathrm{ICH}$ resulted in marked behavioral deficits. The scores of the tests gradually decreased from day 7 to 28 following ICH. Compared with the vehicle-treated group, the rhEPO group showed significantly lower scores in the tests at days 7, 14, 21 and 28 after $\mathrm{ICH}$, suggesting that rhEPO treatment significantly improved the neurological function in these rats from day 7 onwards. To determine whether the rhEPO-mediated behavioral improvements were associated with activation of the PI3K-Akt signaling pathway, the PI3K inhibitor wortmannin was injected prior to the administration of rhEPO. The group receiving co-administration of rhEPO and wortmannin showed significantly higher scores in the two tests at all time-points from day 7 to 28 compared with the group with rhEPO alone. Moreover, no difference was identified between the vehicle-treated group and the rhEPO + wortmannin co-treated group at all time-points, suggesting that inhibiting the activation of the PI3K-Akt signaling pathway abolished the beneficial effect of rhEPO on behavioral outcomes.

rhEPO reduces levels of proinflammatory cytokines in striatal tissue. Proinflammatory cytokine (IL-1 $\beta$, TNF- $\alpha$, 
A

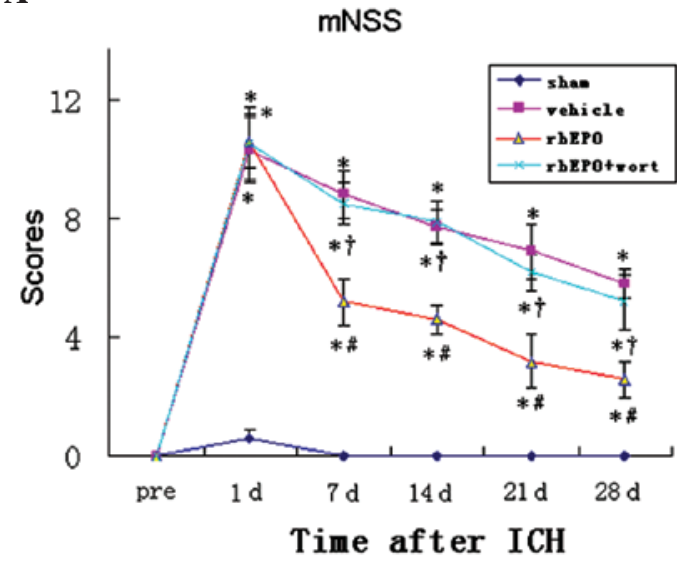

B

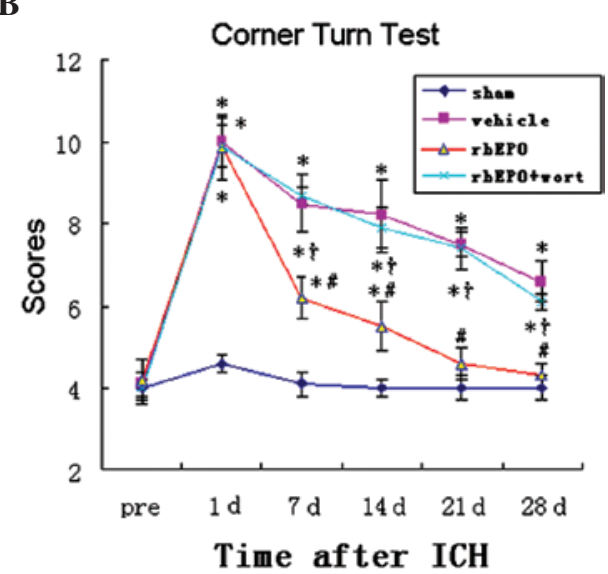

Figure 3. Measurement of neurological function using mNSS and the corner turn test. rhEPO treatment improved functional outcomes following intracerebral hemorrhage. Neurological scores at days 1, 7, 14, 21 and 28 following ICH or sham surgery are shown. (A) mNSS scores and (B) corner turn test. Data are expressed as the mean \pm SEM. ${ }^{*} \mathrm{P}<0.05$ vs. the sham-operated group, ${ }^{\prime \prime} \mathrm{P}<0.05$ vs. the vehicle-treated group and ${ }^{\dagger} \mathrm{P}<0.05$ vs. the rhEPO-treated group ( $\mathrm{n}=6$ per group). mNSS, modified neurological severity score; rhEPO, recombinant human erythropoietin; ICH, intracerebral hemorrhage; wort, wortmannin.

A

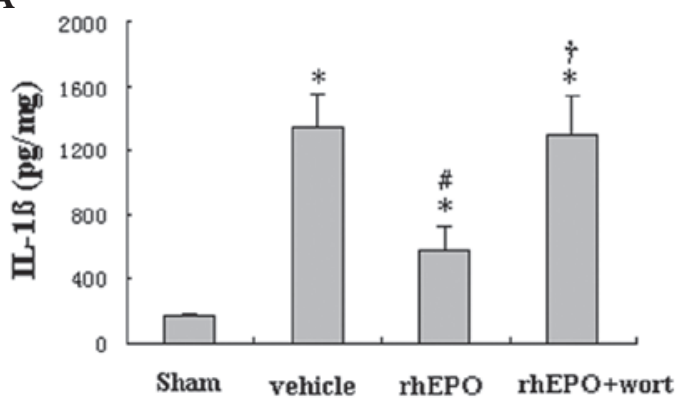

C
B

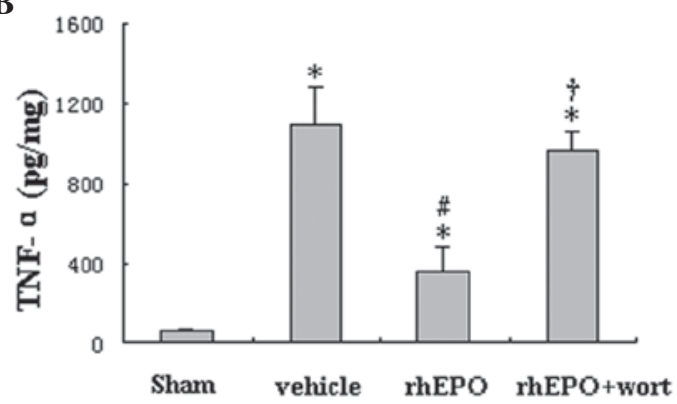

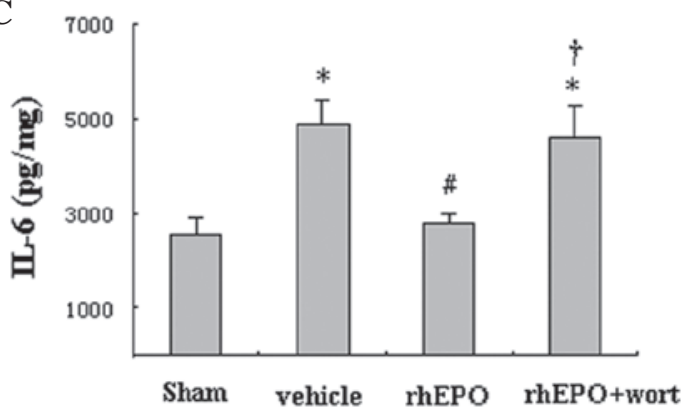

Figure 4. Levels of IL-1 $\beta$, TNF- $\alpha$ and IL-6 in the ipsilateral striatal tissues $24 \mathrm{~h}$ following ICH. The levels of proinflammatory cytokines were measured by an enzyme-linked immunosorbent assay. (A) IL-1 $\beta$, (B) TNF- $\alpha$ and (C) IL- 6 . Values are presented as the mean \pm SEM, ${ }^{2}$ P $<0.05$ vs. the sham-operated group, ${ }^{\#} \mathrm{P}<0.05$ vs. the vehicle-treated group and ${ }^{\dagger} \mathrm{P}<0.05$ vs. the rhEPO-treated group ( $\mathrm{n}=6$ per group). IL-1 $\beta$, interleukin-1 $\beta$; TNF- $\alpha$, tumor necrosis factor- $\alpha$; $\mathrm{ICH}$, intracerebral hemorrhage; rhEPO, recombinant human erythropoietin; wort, wortmannin.

and IL-6) levels in the ipsilateral striatum were determined by ELISA at day 1 following ICH. Compared with the sham-operated rats, vehicle-treated hemorrhagic rats exhibited significantly higher levels of IL-1 $\beta$, TNF- $\alpha$ and IL-6 (Fig. 4A-C, P<0.05), suggesting an ICH-induced inflammatory response. Treatment with rhEPO significantly reversed the ICH-induced upregulation of IL- $1 \beta$ TNF- $\alpha$ and IL-6, reaching that of the sham-operated group. However, wortmannin treatment inhibited the effect of rhEPO on attenuating the inflammatory response. This suggested that the activation of the PI3K-Akt signaling pathway is involved in the rhEPO-mediated inhibition of the inflammatory response following $\mathrm{ICH}$.
rhEPO reduces neuronal apoptosis $24 \mathrm{~h}$ following $\mathrm{ICH}$. Neuronal apoptosis was analyzed in rats from each group ( $\mathrm{n}=6$ per group) $24 \mathrm{~h}$ following ICH or sham operation. Immunoreactivity of NeuN (red) and TUNEL staining (green) were used to identify neuronal apoptosis in the perihematomal area or near the needle tract in the sham animals. Representative images for each group are shown in Fig. 5. Compared with the sham-operated group (Fig. 5A), the vehicle-treated hemorrhagic group (Fig. 5B) showed a decrease in NeuN-positive cells, but significant increases in TUNEL-positive and TUNEL/NeuN double-positive cells (Fig. 5E) in the perihematomal area, suggesting ICH-induced apoptosis and neuronal loss in the injured area. rhEPO treatment restored NeuN-positive cells 

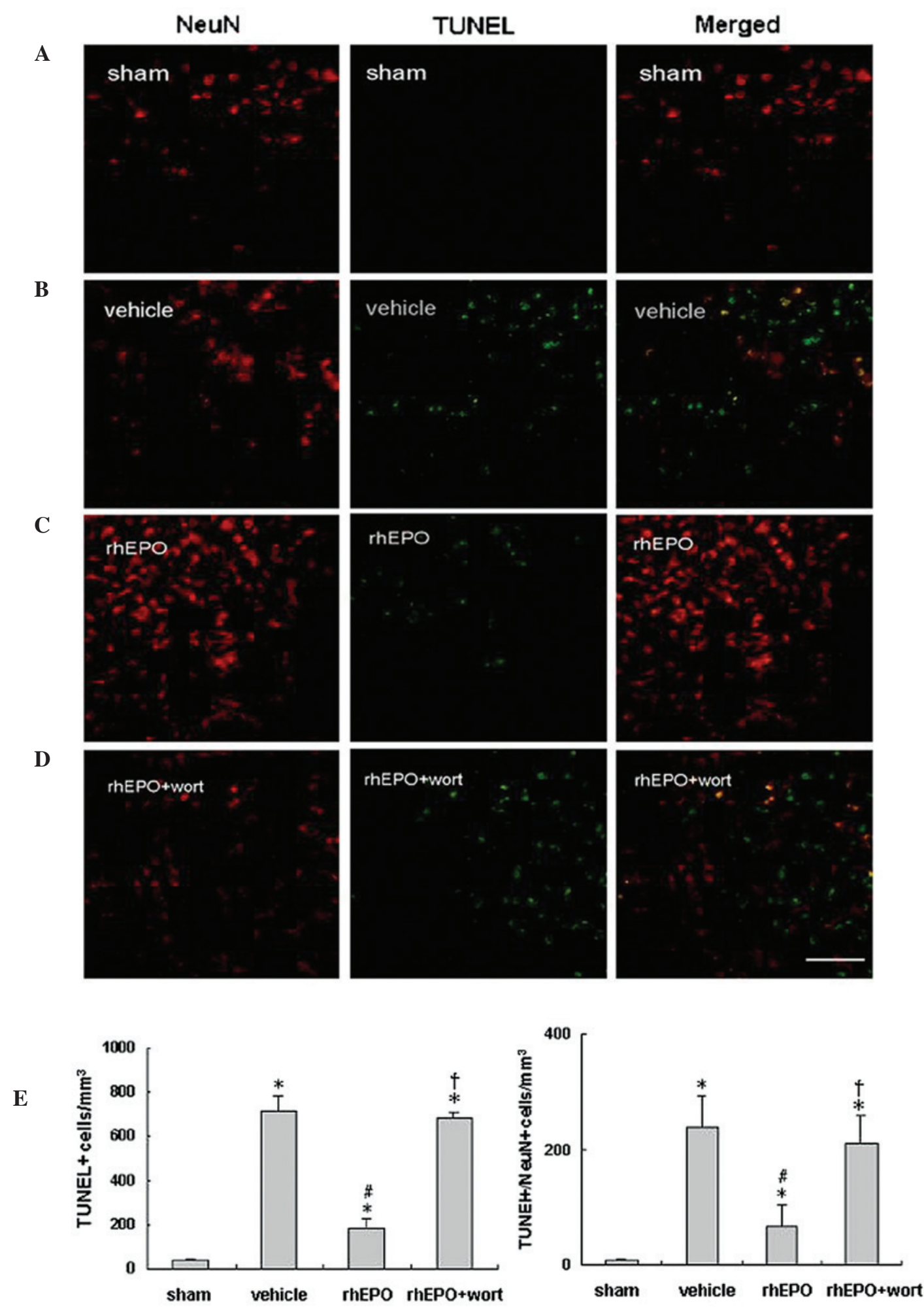

Figure 5. Quantification of neuronal apoptosis with NeuN and double-staining. NeuN staining is shown in red and TUNEL staining in green. (A) Sham, (B) vehicle, (C) rhEPO and (D) rhEPO + wort-treated cells. (E) Quantification of TUNEL-positive and TUNEL/NeuN double-positive cells. " $\mathrm{P}<0.05$ vs. the sham-operated group; ${ }^{*} \mathrm{P}<0.05$ vs. Sham group; ${ }^{\#} \mathrm{P}<0.05$ vs. vehicle-treated group; ${ }^{\circ} \mathrm{P}<0.05$ vs. rhEPO-treated group ( $\mathrm{n}=4$ per group). Scale bar, $50 \mu \mathrm{m}$. TUNEL, terminal deoxynucleotidyl transferase dUTP nick end labeling; wort, wortmannin; rhEPO, recombinant human erythropoietin.

and decreased neuronal apoptosis (Fig. 5C), as shown with significantly decreased TUNEL- and TUNEL/NeuN double-positive cells compared with the vehicle-treated group (Fig. 5E, $\mathrm{P}<0.05$ ). Administration of wortmannin prior to rhEPO treatment abolished the anti-apoptotic effect of rhEPO (Fig. 5C and D; $\mathrm{P}<0.05$ vs. the rhEPO-treated group), and no significant difference was observed between the vehicle- and rhEPO + wortmannin-treated groups $(\mathrm{P}>0.05)$.

rhEPO activates the PI3K-Akt signaling pathway and decreases $p$-GSK-3 $\beta$ expression 24 h after ICH. To further study the involvement of the PI3K-Akt signaling pathway in the 
A
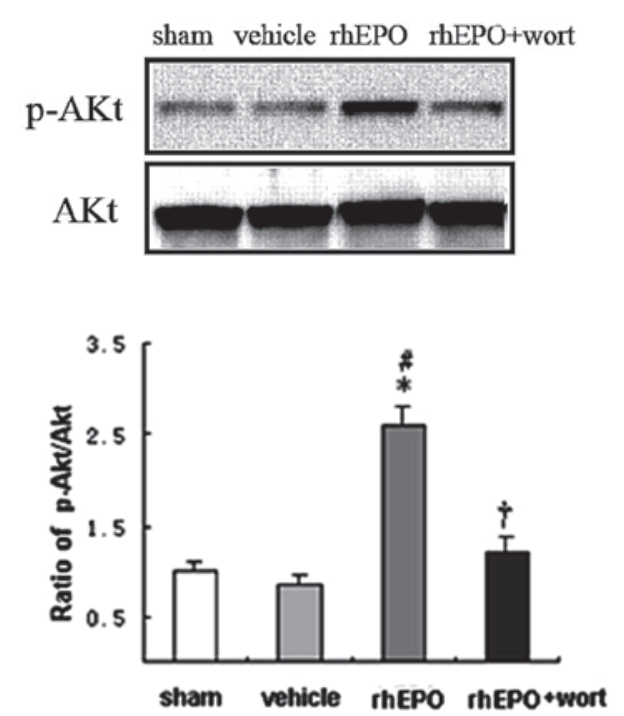

B
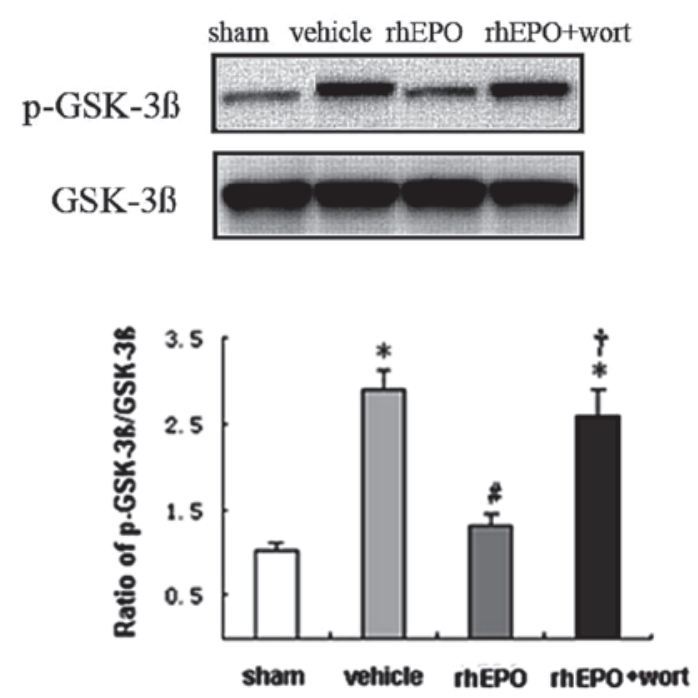

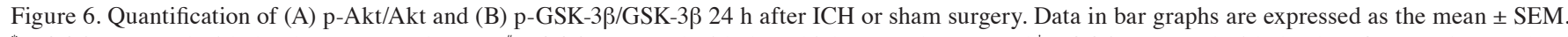
${ }^{*} \mathrm{P}<0.05$ compared with the sham-operated group, ${ }^{*} \mathrm{P}<0.05$ compared with the vehicle-treated group and ${ }^{\dagger} \mathrm{P}<0.05$ compared with the rhEPO-treated group $(\mathrm{n}=6$

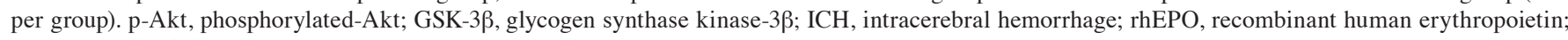
wort, wortmannin.

rhEPO-mediated neuroprotective and anti-apoptotic effects, western blot analysis was conducted. The level of phosphorylated Akt (p-Akt, Ser473) and GSK-3 $\beta$ (p-GSK-3 $\beta$, Tyr9) were quantified $24 \mathrm{~h}$ following ICH. The ratios of $\mathrm{p}-\mathrm{Akt} / \mathrm{Akt}$ and p-GSK-3 $3 /$ GSK-3 $\beta$ were used to indicate a change in the phosphorylation level. The phosphorylation level of Akt showed no change following ICH, but a significant increase after rhEPO treatment (Fig. 6A). By contrast, the phosphorylation level of GSK-3 $\beta$ showed a 2.7 -fold increase $(\mathrm{P}<0.05)$ in response to $\mathrm{ICH}$ and it was reversed to approximately the level of the sham group following rhEPO-treatment (Fig. 6B). Wortmannin pre-treatment abolished the effect of rhEPO on the regulation of the phosphorylation levels of Akt and GSK-3 $\beta$.

\section{Discussion}

ICH remains a serious clinical problem that lacks effective treatment. In the present study, the efficacy of rhEPO was analyzed following hemorrhagic brain injury and the possible signaling pathway involved in the rhEPO-mediated effect was investigated. It was demonstrated that rhEPO treatment following ICH resulted in a significant reduction in brain edema and inhibition of inflammation and apoptosis that consequently led to neurological improvement. Pharmacological intervention using wortmannin suggested that the activation of the PI3K-Akt signaling pathway contributed to the rhEPO-mediated beneficial effect. The results suggested the usage of rhEPO may be a therapeutic modality for ICH injury.

The neuroprotectivity of EPO has been demonstrated in animal and human models under several neurological conditions, including ischemic stroke and traumatic brain injury. This neuroprotective effect may be due to multiple mechanisms, including anti-apoptosis, anti-inflammation, anti-oxidation, angiogenesis and its function as a eurotrophic factor. These effects consequently reduce the ischemic infarct area and neuronal loss, as well as promote cell survival following experimental insult $(27,28)$. In the present study, different measures were combined in an ICH model, which further confirmed that EPO may exert its protective effects through a variety of mechanisms at the cellular and molecular levels and may have the potential to affect short- and long-term clinical outcomes.

ICH initiates prominent inflammatory responses, including the activation of resident glial cells and infiltration of circulating inflammatory cells into the brain $(3,29,30)$. These inflammatory cells produce a number of proinflammatory mediators, such as TNF- $\alpha$, IL-1 $\beta$ and IL-6 (31). TNF- $\alpha$ is associated with neuronal death and inflammation in stroke (32). IL-1 $\beta$ may also be involved in the pathogenesis of ICH, since overexpression of the IL-1 receptor antagonist significantly reduces brain edema induced by the injection of autologous blood or thrombin into the rat striatum (33). This suggests that the release of proinflammatory cytokines following ICH may result in severe secondary damage in neurons, which results in long-term neurological deficits. Brain edema is commonly used as an indicator of brain damage following $\mathrm{ICH}$, and has been shown to be correlated with the release of proinflammatory cytokines. Brain edema is defined as an increase in the water content of brain tissue and is commonly observed during the acute and subacute stages of ICH. Studies have shown that the perihematomal edema is a result of the local inflammatory response and contributes to poor neurological outcomes $(34,35)$. Moreover, anti-inflammatory therapies attenuate brain injury $(33,36)$. In the present study, a rhEPO-mediated reduction of proinflammatory cytokines was observed 1 day post-ICH, which was followed by attenuated edema and recovery of neurological performance. It has previously been shown that rhEPO markedly attenuates glial activation and inhibits leukocyte infiltration in a rat model of middle cerebral artery 
occlusion (37). Therefore, one possible link between rhEPO treatment and the decreased level of proinflammatory cytokines may be rhEPO-mediated inhibition of immune cell activation, possibly through modulating the expression of the EPOR on the surface of these cells. As these immune cells are important sources of inflammatory mediators, attenuated activation results in decreased inflammatory cytokine release and thereby inhibits the propagation of the damaged area (38).

Although the neuroprotectivity of EPO is associated with multiple mechanisms, including anti-inflammation and anti-apoptosis, the molecular mechanisms underlying these effects remain unclear. It has been shown that EPO binding to its receptor prevents neuronal apoptosis, which involves the activation of janus kinase 2 and the nuclear factor- $\kappa \mathrm{B}$ signaling pathways (20). In addition, EPO has also been shown to prevent apoptotic injury through an Akt-dependent mechanism (39). Akt has been observed to prevent cellular apoptotic degradation by inhibiting GSK-3 $\beta$ activity $(40,41)$. To the best of our knowledge, the present study investigated for the first time the involvement of the PI3K-Akt signaling pathway in EPO-induced neuroprotection using the specific PI3K inhibitor wortmannin. Inhibiting the PI3K pathway abolished the neuroprotective effect of EPO, suggesting that the PI3K pathway is essential in EPO-induced protection against ICH. rhEPO treatment significantly increased the protein expression of activated Akt in the ipsilateral hemisphere $24 \mathrm{~h}$ following surgery, which successively reduced the expression of activated GSK-3 $\beta$. Wortmannin treatment itself did not result in damage or exacerbate the neurological outcome (42), and wortmannin administration in combination with rhEPO showed no change in the levels of the respective proteins compared with the vehicle-treated group. These results suggest that rhEPO exerts an anti-apoptotic effect through PI3K-Akt activation and results in GSK-3 $\beta$ inhibition.

In conclusion, rhEPO treatment following ICH is beneficial and improves neurological recovery in a rat ICH model through a variety of cellular mechanisms, including the reduction of edema and the inhibition of inflammation and apoptosis. In addition, the rhEPO-mediated effect involved the activation of the PI3K pathway.

\section{Acknowledgements}

This study was supported by grants from the Natural Science Foundation of Chongqing (grant no. CSTC2012JJA10067). The project was sponsored by the Municipal Educational Commission Foundation of Chongqing (grant no. KJ110309).

\section{References}

1. Ostrowski RP, Colohan AR and Zhang JH: Mechanisms of hyperbaric oxygen-induced neuroprotection in a rat model of subarachnoid hemorrhage. J Cereb Blood Flow Metab 25 554-571, 2005.

2. Lyden PD and Zivin JA: Hemorrhagic transformation after cerebral ischemia: mechanisms and incidence. Cerebrovasc Brain Metab Rev 5: 1-16, 1993.

3. Zeng JS, Zheng P, Xu JF, et al: Prediction of motor function by diffusion tensor tractography in patients with basal ganglion haemorrhage. Arch Med Sci 7: 310-314, 2011.
4. Broderick J, Connolly S, Feldmann E, Hanley D, Kase C, Krieger D, et al: Guidelines for the management of spontaneous intracerebral hemorrhage in adults: 2007 update: a guideline from the American Heart Association/American Stroke Association Stroke Council, High Blood Pressure Research Council, and the Quality of Care and Outcomes in Research Interdisciplinary Working Group. Stroke 38: 2001-2023, 2007.

5. Daverat P, Castel JP, Dartigues JF and Orgogozo JM: Death and functional outcome after spontaneous intracerebral hemorrhage. A prospective study of 166 cases using multivariate analysis. Stroke 22: 1-6, 1991.

6. Cotena S, Piazza O and Tufano R: The use of erythropoietin in cerebral diseases. Panminerva Med 50: 185-192, 2008.

7. Li L, Jiang Q, Ding G, et al: MRI identification of white matter reorganization enhanced by erythropoietin treatment in a rat model of focal ischemia. Stroke 40: 936-941, 2009.

8. Ruscher K, Freyer D, Karsch M, et al: Erythropoietin is a paracrine mediator of ischemic tolerance in the brain: evidence from an in vitro model. J Neurosci 22: 10291-10301, 2002.

9. Sirén AL, Fratelli M, Brines M, et al: Erythropoietin prevents neuronal apoptosis after cerebral ischemia and metabolic stress. Proc Natl Acad Sci USA 98: 4044-4049, 2001.

10. Celik M, Gökmen N, Erbayraktar S, et al: Erythropoietin prevents motor neuron apoptosis and neurologic disability in experimental spinal cord ischemic injury. Proc Natl Acad Sci USA 99: 2258-2263, 2002.

11. Hwang J, Huh J, Kim J, Jeon Y, Cho S and Han S: Pretreatment with erythropoietin attenuates the neurological injury after spinal cord ischaemia. Spinal Cord 50: 208-212, 2012.

12. Xiong Y, Mahmood A, Meng Y, Zhang Y, Qu C, Schallert T and Chopp M: Delayed administration of erythropoietin reducing hippocampal cell loss, enhancing angiogenesis and neurogenesis, and improving functional outcome following traumatic brain injury in rats: comparison of treatment with single and triple dose. J Neurosurg 113: 598-608, 2010.

13. Xiong Y, Mahmood A, Lu D, et al: Histological and functional outcomes after traumatic brain injury in mice null for the erythropoietin receptor in the central nervous system. Brain Res 1230: 247-257, 2008.

14. Chen G, Zhang S, Shi J, Ai J and Hang C: Effects of recombinant human erythropoietin (rhEPO) on JAK2/STAT3 pathway and endothelial apoptosis in the rabbit basilar artery after subarachnoid hemorrhage. Cytokine 45: 162-168, 2009.

15. Grasso G, Buemi M, Alafaci C, et al: Beneficial effects of systemic administration of recombinant human erythropoietin in rabbits subjected to subarachnoid hemorrhage. Proc Natl Acad Sci USA 99: 5627-5631, 2002.

16. Tseng MY, Hutchinson PJ, Richards HK, et al: Acute systemic erythropoietin therapy to reduce delayed ischemic deficits following aneurysmal subarachnoid hemorrhage: a Phase II randomized, double-blind, placebo-controlled trial. J Neurosurg 111: 171-180, 2009.

17. Chau M, Chen D and Wei L: Erythropoietin attenuates inflammatory factors and cell death in neonatal rats with intracerebral hemorrhage. Acta Neurochir Suppl 111: 299-305, 2011.

18. Seyfried DM, Han Y, Yang D, Ding J and Chopp M: Erythropoietin promotes neurological recovery after intracerebral hemorrhage in rats. Int J Stroke 4: 250-256, 2009.

19. Grasso G, Graziano F, Sfacteria A, et al: Neuroprotective effect of erythropoietin and darbepoetin alfa after experimental intracerebral hemorrhage. Neurosurgery 65: 763-770, 2009.

20. Lee ST, Chu K, Sinn DI, et al: Erythropoietin reduces perihematomal inflammation and cell death with eNOS and STAT3 activations in experimental intracerebral hemorrhage. J Neurochem 96: 1728-1739, 2006.

21. Seyfried D, Han Y, Lu D, Chen J, Bydon A and Chopp M: Improvement in neurological outcome after administration of atorvastatin following experimental intracerebral hemorrhage in rats. J Neurosurg 101: 104-107, 2004.

22. Duris K, Manaenko A, Suzuki H, Rolland WB, Krafft PR and Zhang JH: $\alpha 7$ nicotinic acetylcholine receptor agonist pnu-282987 attenuates early brain injury in a perforation model of subarachnoid hemorrhage in rats. Stroke 42: 3530-3536, 2011.

23. Wishka DG, Walker DP, Yates KM, Reitz SC, Jia S, Myers JK, et al: Discovery of N-[(3R)-1-azabicyclo[2.2.2]oct-3-yl]furo[2,3c]pyridine-5-carboxamide, an agonist of the alpha7 nicotinic acetylcholine receptor, for the potential treatment of cognitive deficits in schizophrenia: synthesis and structure--activity relationship. J Med Chem 49: 4425-4436, 2006. 
24. Aronowski J and Hall CE: New horizons for primary intracerebral hemorrhage treatment: experience from preclinical studies. Neurol Res 27: 268-279, 2005.

25. Chen J, Li Y, Wang L, et al: Therapeutic benefit of intravenous administration of bone marrow stromal cells after cerebral ischemia in rats. Stroke 32: 1005-1011, 2001.

26. Ma Q, Manaenko A, Khatibi NH, Chen W, Zhang JH and Tang J: Vascular adhesion protein-1 inhibition provides antiinflammatory protection after an intracerebral hemorrhagic stroke in mice. J Cereb Blood Flow Metab 31: 881-893, 2011.

27. Del Bigio MR, Yan HJ, Buist R and Peeling J: Experimental intracerebral hemorrhage in rats. Magnetic resonance imaging and histopathological correlates. Stroke 27: 2312-2319, 1996.

28. van der Kooij MA, Groenendaal F, Kavelaars A, et al: Neuroprotective properties and mechanisms of erythropoietin in in vitro and in vivo experimental models for hypoxia/ischemia. Brain Res Rev 59: 22-33, 2008.

29. Loftspring MC, McDole J, Lu A, Clark JF and Johnson AJ: Intracerebral hemorrhage leads to infiltration of several leukocyte populations with concomitant pathophysiological changes. J Cereb Blood Flow Metab 29: 137-143, 2009.

30. Wang J: Preclinical and clinical research on inflammation after intracerebral hemorrhage. Prog Neurobiol 92: 463-477, 2010.

31. Hanisch UK: Microglia as a source and target of cytokines. Glia 40: 140-155, 2002

32. Allan SM and Rothwell NJ: Cytokines and acute neurodegeneration. Nat Rev Neurosci 2: 734-744, 2001.

33. Masada T, Hua Y, Xi G, Yang GY, Hoff JT and Keep RF: Attenuation of intracerebral hemorrhage and thrombin-induced brain edema by overexpression of interleukin-1 receptor antagonist. J Neurosurg 95: 680-686, 2001.

34. Xi G, Keep RF and Hoff JT: Mechanisms of brain injury after intracerebral haemorrhage. Lancet Neurol 5: 53-63, 2006.
35. Zazulia AR, Diringer MN, Derdeyn CP and Powers WJ: Progression of mass effect after intracerebral hemorrhage. Stroke 30: 1167-1173, 1999.

36. Jung KH, Chu K, Jeong SW, et al: HMG-CoA reductase inhibitor, atorvastatin, promotes sensorimotor recovery, suppressing acute inflammatory reaction after experimental intracerebral hemorrhage. Stroke 35: 1744-1749, 2004.

37. Villa P, Bigini P, Mennini T, Agnello D, et al: Erythropoietin selectively attenuates cytokine production and inflammation in cerebral ischemia by targeting neuronal apoptosis. J Exp Med 198: 971-975, 2003.

38. Liu X, Shen J, Jin Y, Duan M and Xu J. Recombinant human erythropoietin (rhEPO) preconditioning on nuclear factor-kappa B (NF-kB) activation \& proinflammatory cytokines induced by myocardial ischaemia-reperfusion. Indian J Med Res 124: 343-354, 2006.

39. Belayev L, Khoutorova L, Zhao W, et al: Neuroprotective effect of darbepoetin alfa, a novel recombinant erythropoietic protein, in focal cerebral ischemia in rats. Stroke 36: 1071-1076, 2005.

40. Brines ML, Ghezzi P, Keenan S, et al: Erythropoietin crosses the blood-brain barrier to protect against experimental brain injury. Proc Natl Acad Sci USA 97: 10526-10531, 2000.

41. Valerio A, Bertolotti P, Delbarba A, et al: Glycogen synthase kinase-3 inhibition reduces ischemic cerebral damage, restores impaired mitochondrial biogenesis and prevents ROS production. J Neurochem 116: 1148-1159, 2011.

42. Yano S, Morioka M, Fukunaga K, et al: Activation of Akt/protein kinase $\mathrm{B}$ contributes to induction of ischemic tolerance in the CA1 subfield of gerbil hippocampus. J Cereb Blood Flow Metab 21: 351-360, 2001. 